\title{
Role of Mesenchymal Stem Cell Based Therapies in MDR/ XDR TB and Co-Morbidities
}

Ranganathan N lyer ${ }^{1 *}$, Eswara Prasad Chelluri ${ }^{2}$ and Lakshmi Kiran Chelluri ${ }^{3}$

${ }^{1}$ Department of Clinical Microbiology, Global Hospitals, Hyderabad, India

${ }^{2}$ Department of Respiratory Medicine, Shadan Institute of Medical Sciences, Hyderabad, India

${ }^{3}$ Department of Transplant Immunology and Stem Cell Lab, Global Hospitals, Hyderabad, India

\begin{abstract}
Multi drug resistant tuberculosis in patients with co-morbidities such as diabetes mellitus, HIV and other respiratory disorders is a major challenge for intervention. There is an upsurge in these cases in South East Asia and Africa. Failed therapy protocols of MDR/XDR tuberculosis has placed a demand for new therapeutic strategies. Immunotherapy has been in vogue for a long time with negligible success rates. Co-morbidities in patients compound and complicate the existing treatment options which necessitate a search for amenable and comprehensive treatment protocols. One such approach is mesenchymal stem cell adjunct therapy $t$ for MDR/XDR- TB. Whilst Mesenchyma stem cells are an interesting option, inherent problems related to the dose and timing of administration warrant extensive clinical research before their acceptance in clinical management protocols. The mechanism of action of these cells is illunderstood at the present time and awaits further supportive experimental data. It appears that stem cell therapy could be an option to complement existing therapeutic protocols for the management of drug resistant tuberculosis. The current review deals with the mechanistic and surrogate markers of therapeutic benefit of such novel approaches.
\end{abstract}

Keywords: MDR/XDR TB; Mesenchymal stem cells; Cytokines; Therapeutic modality; Co-morbidities

\section{Introduction}

Multi-Drug Resistant (MDR)/Extremely Drug Resistant (XDR) tuberculosis is an increasing disease burden worldwide with a high prevalence in Eastern Europe, South Africa and South East Asia [1]. Treatment strategies including directly observed Treatment short course (DOTS), DOTS-Plus, recombinant human interleukin -2(rhuIL2) by aerosol treatment and recombinant interferon-gamma, have met with varied degrees of success [2-4]. It is a proven fact that the host immune profile plays a vital role in disease susceptibility. Numerous studies have documented the role of inflammatory cytokines in the depressed immune profile of the host. It appears from various studies, that (T helper) Th counter-regulation holds the key, to turn the pro-inflammatory responses to anti-inflammatory responses. In this context, several adjunct immunotherapeutic strategies have also been tried for their efficacy at various stages of active tuberculosis [5]. Mesenchymal stem cells (MSC's) being immune-modulatory, have been an ideal choice as adjunct therapy in MDR/XDR tuberculosis, particularly in patients with co-morbidities.

\section{Mesenchymal Stem Cells}

Mesenchymal stem cells have been isolated from various tissue sources such as blood, bone marrow and adipose tissue in adults, umbilical cord, chorionic villi and amniotic fluid and somatic cell iPSCs which are induced pluripotent stem cells (Figure 1).

MSCs have demonstrable properties of differentiating into three lineages of the germ layers, the endoderm, ectoderm and the mesoderm. They are hypo-immunogenic in nature and negative to HLA-DR. They are inert to co-stimulatory molecules in allogenic applications, thereby proving to be a distinct cell candidate for immune modulation. MSCs are currently being investigated in the reversal of acute GvHD, chronic inflammatory diseases and autoimmune diseases, with varied success [6,7].

The presence of multipotent stromal cells was first suggested by a German Pathologist Cohnheim. These stromal cells constitute 0.001 to $0.01 \%$ of the entire population of bone marrow nucleated

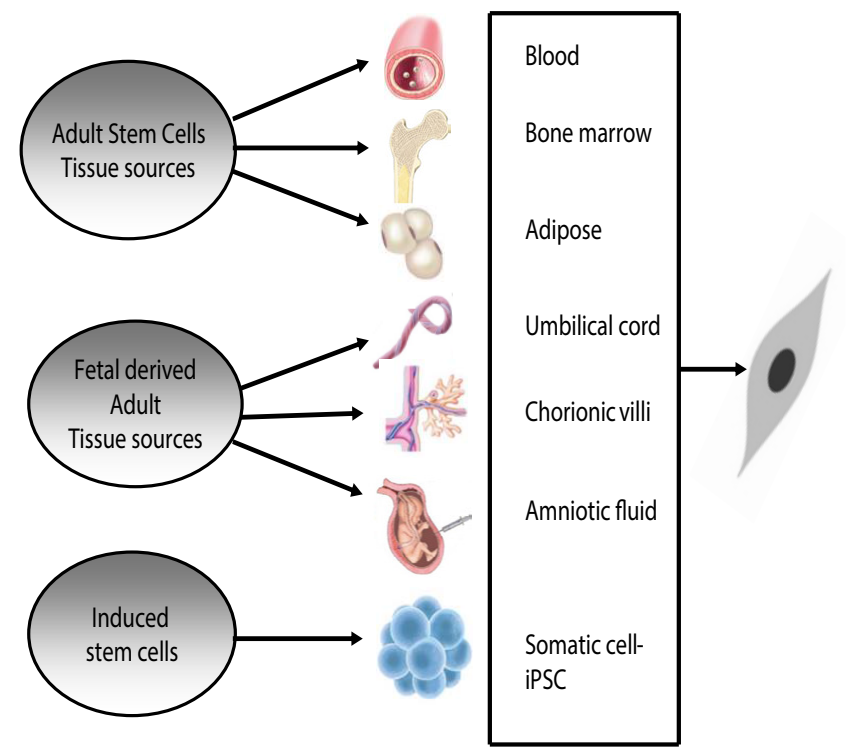

Figure 1: Mesenchymal stem cells can be sourced from various adult tissue sources such as peripheral blood, bone marrow, adipose tissue from omentum; fetal tissue sources from umbilical cord blood, chorionic villi, amniotic fluid; induced somatic cells such as induced pluripotent stem cells [iPSC].

*Corresponding author: Dr. Ranganathan lyer MD FRCPath DNB DPB, Consultant Clinical Microbiologist, Global Hospital, Lakdi- ka- Pul, Hyderabad- 500004, India, Tel: +91-40-30244505; E-mail: ranganathaniyer@yahoo.com

Received April 22, 2015; Accepted May 27, 2015; Published May 29, 2015

Citation: Iyer RN, Chelluri EP, Chelluri LK (2015) Role of Mesenchymal Stem Cell Based Therapies in MDR/ XDR TB and Co-Morbidities. J Stem Cell Res Ther 5: 284. doi:10.4172/2157-7633.1000284

Copyright: ( 2015 lyer RN, et al. This is an open-access article distributed under the terms of the Creative Commons Attribution License, which permits unrestricted use, distribution, and reproduction in any medium, provided the original author and source are credited. 
cells. According to the International Society for Cellular Therapy, the minimum criteria to define cells as stem cells are: $[8,9]$

1. They adhere to plastic under standard culture conditions.

2. They are positive for CD105, CD73, and CD90 and negative for hematopoietic stem cell markers such as CD34, CD45, and CD11a CD19 and HLA-DR.

3. Under specific stimuli, they differentiate into chondrocytes, myocytes, osteocytes and adipocytes in vitro (Figure 2).

\section{The Immuno-pathology of Tuberculosis}

The cellular arm of immunity plays a vital role in the host resistance to infection with $M$. tuberculosis. The ability of the host immune response to contain and prevent the spread of the tubercle bacilli determines the robustness of the host response. The two cytokines that play a key role in this process are Interferon - gamma (IFN- $\gamma$ ) and Tumour Necrosis factor- alpha (TNF- $\alpha$ ). Of these two cytokines, IFN- $\gamma$ helps contain bacterial replication and prevents a destructive immune response to the infection. It has been observed by Chelluri et al. that mesenchymal stem cells have the propensity to respond in culture supernatants of $M$. tuberculosis in a dose dependent manner [10]. The IFN- $\gamma$ produced by $\mathrm{T}$ lymphocytes stimulates macrophages to produce toxic nitric oxide intermediates which are inhibitory to tubercle bacilli. The tubercle bacilli, engulfed by macrophages remain dormant but are not killed for many years. This forms a Th1 response to the infection. Hence CD $4+\mathrm{T}$ lymphocytes which do not express IFN- $\gamma$ retain antimicrobial activity, though they lose the ability to suppress the inflammatory response. Another cytokine that correlates with IFN- $\gamma$ is IL- 17 that recruits plenty of neutrophils to the site of infection. Increased number of these cells indicates a failed Th1 response with reduced levels of IFN- $\gamma$. It is hypothesized that the IFN- $\gamma$, when administered, may elaborate Il-10 that increases the recruitment of lymphocytes and reduces the number of neutrophils to the site of the infection. This in turn, reduces the deleterious effects of continued presence of neutrophils such as enhanced tissue destruction and limited immune mediate lung injury with an improved time to clearance of $M$. tuberculosis infection [11].

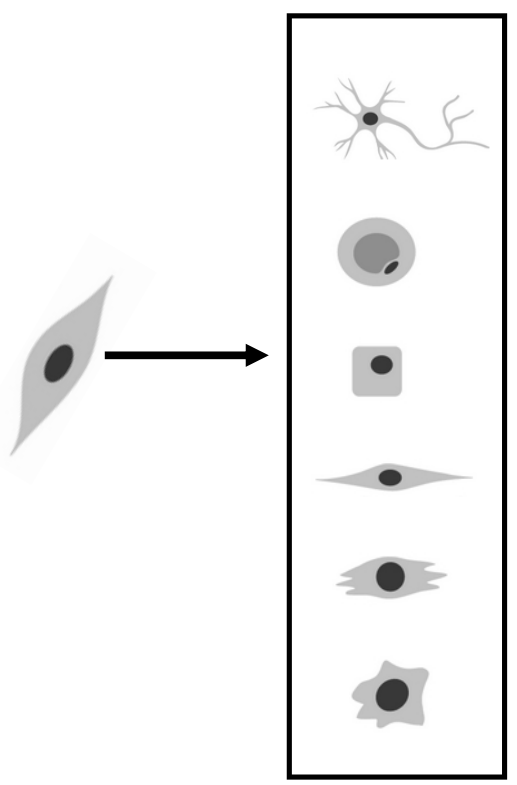

Neuron

Adipocyte

Osteoblast

Vascular smooth muscle cell

Myocyte

Chondrocyte

Figure 2: Mesenchymal stem cells have tri-lineage potential to differentiate i.e., ectoderm, endoderm and mesoderm.

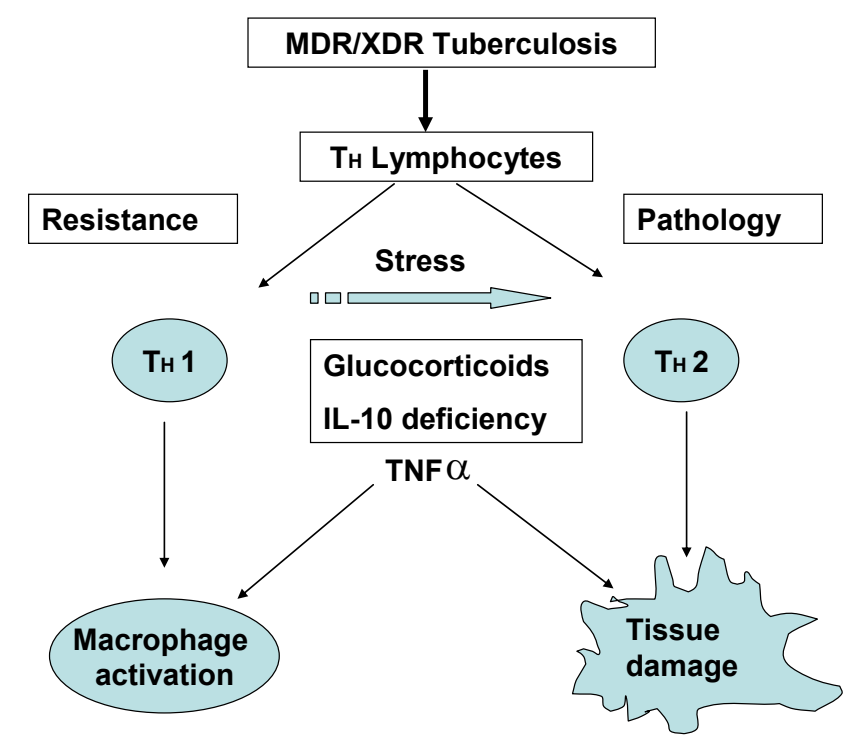

Figure 3: Protective immunity requires a Th1 response, and tumour necrosis factor (TNF) is needed as an additional, macrophage activating factor. With a mixed Th1-Th2 cytokine response, TNF becomes toxic and mediates the gross tissue destruction characteristic of active, progressive tuberculosis. Stress and corticosteroids tend to drive newly recruited T cells towards Th2 responses. The protective Th1 immunity, a characteristic feature of granuloma is regulated by two mechanisms firstly (1) IFN-gamma which is essential for granuloma formation, may limit the uncontrolled expansion of mycobacteriareactive $T$ cells. Secondly, endogenous $\mathrm{IL}-10$, produced during mycobacterial infections, can reduce IL-12 production from mycobacteria infected DC's, migration of DC's to draining lymph nodes and the expansion of IFN-gamma secreting T-cells. However, the effective dose of IFN- $\gamma$ and timing is yet to be determined.

Thus, there is a beneficial role of IFN- $\gamma$ in preventing increased tissue destruction in tuberculosis and maintaining a balance between the organisms and the enhanced $\mathrm{T}$ cell response in the same host. However all these are dependent on the dose and the timing of the IFN- $\gamma$ administration which is yet to be unraveled (Figure 3).

\section{Possible Mechanisms of Mesenchymal Stem Cells in MDR/XDR-Tb}

Mesenchymal stem cells play a key role in tissue regeneration and immune function modulation - a consequence of complex cell - cell interactions. Mesenchymal stem cells constitutively express soluble immunomodulatory factors such as macrophage stimulating factor, Prostaglandin E2 (PGE2), Hepatocyte growth factor (HGF) and IL-10. IL-10 is a dichotomously functioningcytokine with immunosuppressive and/or immunostimulatory properties. Current reports have shown that tissue specific mesenchymal cells can modify dendritic cell (DC) function demonstrated by an altered capacity to interact with $\mathrm{T}$ cells and induce tolerance or $\mathrm{T}$ cell unresponsiveness. Mycobacteria may use this as a strategy to promote immune deviation towards less effective $\mathrm{T}$ cell responses. It is imperative to understand the underlying mechanisms of host responses and their influence on DC activation. This will aid in designing new therapeutic interventions and vaccines to combat resistant mycobacterial infections. The constitutive secretion of soluble immunomodulators by MSC's is an added advantage to counter single cytokine strategies for therapy such as anti-TNF-alpha, rhu IL-2, IFN-gamma etc. They address the local inflammatory milieu in totality, countering the anti-inflammatory cytokine secretions to balance the Th1/Th2 responses. 


\section{Tb /MDR/XDR infection}

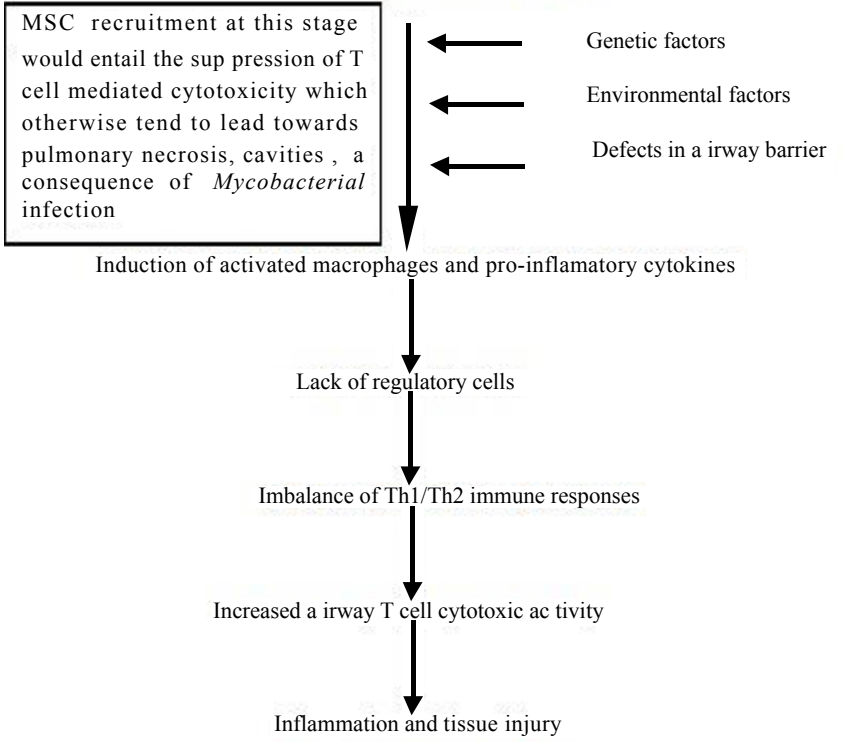

Figure 4: IFN- and TNF- $\alpha$ are the two critical cytokines in counter-regulating the Th imbalance influenced by various external stimuli in the innate and adaptive immune profiles. Mesenchymal stem cell therapy as an adjunct in the treatment of multi-drug resistant/extremely drug resistant tuberculosis cases may help alleviate the cascade of deleterious events and improve the quality of life.

Furthermore, it is speculated that the IL-10 released from mesenchymal stem cell, along with macrophage stimulatory factors, PGE2, and HGF would antagonize the IL-12 and TNF-alpha secreted by $\mathrm{M}$. Tb. These actions along with modification of DC function may serve to make the immune responses favorable to host resistance and down-regulate host susceptibility. The macrophage stimulating factor produced by mesenchymal stem cells would either increase the number of healthy macrophages or enhance phagocytosis by the activated macrophages in the infection stage and inhibit progress to inflammation and subsequent tissue damage (Figure 4). Further, the process may help develop the tolerant phenotype. The interaction between MSC-T cells, MSC-DC1 and DC2 may help us understand the $\mathrm{T}$ cell mediated pathways.

In recent years, morphologically similar cells, not identical to bone marrow stem cells have been shown to originate from the stromal fraction of connective tissue from several organs including adipose tissue, liver and muscle [12]. Hence, it is important to remember that the origin of stem cells could well determine their ultimate fate and functional features. The essential difference between the MSC's obtained from non-marrow sources and those obtained from the bone marrow, is that the latter are capable of a tri-lineage differentiation potential (30\% of the clones), with some of the others exhibiting a bilineage potential, while representatives of the former category do not have this potential. Most of the former groups of cells are said to be bipotent and some of them unipotent [13].

\section{MSC Interaction with Immune Cells}

All mesenchymal stem cells have immunomodulatory characteristics, including inhibition of $\mathrm{T}$ cells proliferation in vitro which was among the first to be observed [14]. These stem cells are known to be immunosuppressive, interacting with other immune cells under the influence of pro-inflammatory cytokines. They may also secrete cytokines which have a suppressive effect [15]. This immunosuppressive potential is not immunologically restricted based on their origin. The degree of immune-suppression is believed to be dose dependent, with higher doses posing an inhibitory effect on T cell proliferation [16]. The following mechanisms of interaction of MSC's with immune cells is noted [17]:

a. They inhibit characteristics of NK (Natural killer) cells, such as their proliferation and cytotoxicity and their ability to produce cytokines via the elaboration of PGE2 (Prostaglandin E2) and indolamine 2,3-dioxygenase (IDO) .

b. Inhibit the differentiation of monocytes into immature myeloid dendritic cells (DC's), production of TNF (tumour necrosis factor) by the DC's and convert mature DC's into immature DC's.

c. Increase the production of IL-10 by plasmacytoid DC's.

d. Release several molecules including PGE2, TGF $\beta-1$ and IDO thereby inhibiting CD4 $\mathrm{T}$ cell function.

e. Promote the formation of regulatory $\mathrm{T}$ cells ( $\mathrm{T}$ regs). This is a subpopulation of $\mathrm{T}$ cells that help prevent tissue damage and associated pathological changes in the lungs and liver. They contain unrestricted expansion of effector T cell populations. They are identified by the co- expression of CD4 and CD25 antigens and production of regulatory cytokines such as IL-10 and TGF- $\beta[18]$.

f. They inhibit B cell function with the help of soluble factors and cell to cell contact.

g. They inhibit the respiratory burst in neutrophils.

Overall, the main interaction with immune cells may be summarized by their modulation of cytokine production by DC Th1 and DC Th2 cells, blocking the maturation of antigen presenting cells (APC's) and increasing the number of Tregs CD4 CD25 positive cells in a mixed population of lymphocytes.

In recent times, MSCs are thought to be actively involved in repair of tissues [19]. Their migration to and differentiation at the site of tissue damage are important steps in tissue repair. They have the ability to secrete cytokines and growth factors at the site of tissue injury and inflammation which contributes significantly to their therapeutic potential [20]. The therapeutic benefits are related to certain features of MSC's such as, their anti-scarring, angiogenic, anti- apoptotic properties, and the fact that they are immunospuuressive for $\mathrm{T}$ cells and other immune cells as well as their regenerative capacity [21]. All these together may contribute to altering acute and chronic inflammatory responses in addition to enhancing the host regenerative capacity [22]. The production of cytokines and their actions are said to be dependent on their location, the niche and severity of the injury.

\section{MSCs in TB and Other Infections}

It is speculated that the ability of MSC's to interact with the internal milieu at the site of the tissue injury may influence their therapeutic potential. . This implies that systemically infused mesenchymal stem cells would repair and regenerates injured tissue at the site directly or influence the host immune system to affect repair [23]. The action of these stem cells on the lung tissue at sites of inflammation would be to tone down the inflammatory response and render it insusceptible to inflammation. In the event of pulmonary fibrosis, they may have a role to reverse the deposition of collagen and to remove deposited collagen. 
The application of MSC's in chronic pulmonary disorders rests on maintaining a balance between the extent of pulmonary fibrosis and the ongoing parenchymal inflammation.

Mesenchymal stem cells are non haematopoeitic stem cells which have emerged as a therapeutic modality in many inflammatory disorders. Their potential has been exploited in cell therapy using a number of strategies such as systemic infusions for the treatment of rheumatoidarthritis, Hemophilia B, Type-1 diabetes mellitus, Graft Versus Host Disease (GvHD), acute myocardial infarction, multiple sclerosis, Crohn's disease and Systemic Lupus erythematosus (SLE) $[24,25]$. They have been used to repair tissue in acute lung injury due to any cause such as COPD (Chronic Obstructive Pulmonary Disease), pulmonary hypertension, asthma and allergy and fibrotic lung disorders [24]. It is found that these stem cells are recruited to the site of infection in pulmonary tuberculosis and form a rim of cells at the periphery of a granuloma. At the periphery, they exert their immunosuppressive effects which could form the basis of immunotherapy in the future. They are thought to express the stem cell marker Sca-1. The mechanism by which they migrate to the periphery of a granuloma or any damaged tissue site in the body and the reason for their survival is not clearly understood due to the lack of reliable tracing markers [25]. It is also known that the immunosuppressive effects of mesenchymal stem cells are mediated by their interaction with IFN- $\gamma$ along with TNF- $\alpha$ and IL1. This is akin to them being licensed to be immunosuppressive. This could be extrapolated to the fact that stem cells are best active when administered after the onset of inflammatory diseases. Interaction and treatment of stem cells with inflammatory cytokines may also help enhance their therapeutic efficacy. This is well brought out in a mouse model with GvHD induced disease [26].

When recruited to the periphery of a granuloma, they elaborate nitric oxide (NO) which in turn suppresses the $\mathrm{T}$ cells within the granuloma. Thus, the granuloma harbors the M. tuberculosis organisms at the centre of the granuloma and the mesenchymal stem cells at the periphery $\mathrm{NO}$ also induce and enhances $\mathrm{T}$ cell apoptosis, thus contributing to the immunosuppressive effect [27]. NO is unique as $\mathrm{t}$ it is active when it is in close proximity to the cells producing it. The immune-suppression reduces as one move away from the granuloma [28].

$\mathrm{NO}$ is an important mycobactericidal agent, thereby increasing the host resistance to tuberculosis. NO produced by the stem cells is in close proximity to the mycobacteria as well as the $\mathrm{T}$ cells, an environment conducive to its production. Thus, stem cells by virtue of their NO production, maintain a dynamic equilibrium between the mycobacteria on the one hand and the enhanced cellular immune response induced by the $\mathrm{T}$ cells on the other $[29,30]$.

Whilst NO has always been found to have inhibitory effects on $\mathrm{T}$ cells at the site of a tuberculous granuloma, they also are found to induce Fox P3+ regulatory $\mathrm{T}$ cells (also called Tregs) from a common pool of CD4 T cells during M. tuberculosis infection. A study from the All India Institute of Medical Sciences (AIIMS) did look at the ability of $M$. tuberculosis to evade host immunity by recruiting mesenchymal stem cells to the site of infection. The doubt remains whether this activity could inhibit $\mathrm{T}$ cells and promote susceptibility to M. tuberculosis infection [31]. The authors in their study found that mesenchymal stem cells did induce Tregs to establish T cell tolerance during $M$. tuberculosis infection. This induction and expansion of Tregs has previously been reported to occur during progression of tuberculosis as a disease in human beings [32].

The capacity to exert an immunosuppressive effect requires the MSC's to be in physical contact with T cells. Due to this property, they do not participate in a generalized immunosuppression, but do exert a localized immunosuppressive effect that inhibits the destructive activity caused by unlimited $\mathrm{T}$ cell activation. Hence it is thought that the mesenchymal stem cells could be a potential immunotherapeutic target for the treatment of tuberculosis $[33,34]$.

\section{MSC's in MDR/XDR with Co-morbidities}

HIV infection complicated by MDR/ XDR tuberculosis calls for a new approach that is non-toxic, less expensive with a promise to improve the quality of life. The present drug regime has several limitations. Skrahin et al., in their recent series have published data of thirty patients who suffered MDR tuberculosis and were infused with mesenchymal stem cells in addition to $h$ conventional anti- tuberculosis therapy with susceptibility testing. Of the 30 patients, sixteen cleared their infection after six months of infusion with stem cells, while the other fourteen demonstrated mild adverse reactions such as high cholesterol, nausea and lymphopenia or diarrhoea [35]. This study imbues us to consider adjunct MSC therapy to the existing therapeutic protocols. Whilst this shows promise, more work and data are needed in this area.

\section{MSC Trials in Other Lung Diseases}

A list of pulmonary disorders with the potential application of mesenchymal stem cells and the status of their application are shown in the Table 1 .

The Skrahin study used autologous mesenchymal stem cells based on the POSEIDON trial in cardiomyopathies. Mesenchymal stem cells used in many clinical trials range between $0.5-3.0 \times 10^{6}$ cells per $\mathrm{kg}$ [37]. There is high variability in the yield of MSCs amongst donors one of them being decline in age. Their study did not show any suppressed peripheral T-cell responses due to MSC infusion, circumventing the risk of increased host immune deviation responses. Timing and dose is a pertinent factor that warrants case controlled Phase-II clinical trials. They propose that MSC infusion in cases of MDR/XDR Tb with co-morbidities such as HIV, Hep B and C may worsen the immune suppression leading to more complicated infections. Hence, MSC infusion in such cases demands a more rigorous approach [35].

In summary, it is felt at this juncture that, there is a role for mesenchymal stem cells as a locally acting immunosuppressive therapeutic modality. This would help contain the mycobacterial infection and also restrict the cellular (delayed type hypersensitivity) DTH response to the tubercle bacilli. The exact mechanism of action of these stem cells is partly worked out and chemicals such as NO are believed to play a role. However, more work needs to be done in this area. Another perplexing issue is the timing of administration of the stem cells vis-à-vis the inflammatory response and the dose of the same

\begin{tabular}{|c|c|c|c|c|}
\hline S. No & Disease condition & Mechanism proposed & The animal model \\
\hline 1 & MDR/XDR-Tb & Adjunct therapy & Autologous open label Phase-I safety trial & [35] \\
\hline 2 & Bacterial diseases & Immunosuppressive and anti-bacterial effects of MSCs & Animal model \\
\hline 3 & Broncho-pulmonary dysplasia & Reparative and regeneration & Animal model \\
\hline
\end{tabular}

Table 1: Mesenchymal Stem Cell clinical trials in Pulmonary Tb,MDR/XDR-Tb. 
to an individual patient, as an excess could also lead to deleterious effects. Medical research in the future will hopefully give us an insight into these aspects of mesenchymal stem cells and the role they have to play in containing the effects.

\section{Perspective}

It is too early to deduce the exact role of mesenchymal stem cell therapy as an adjunct in MDR/ XDR TB with co-morbidities. However this can be a novel therapeutic intervention in patient management. It appears that MSC's can mitigate the severity of the disease. It is to be seen whether further research insights would help us understand the underlying mechanistic pathways for the comfort of safety and efficacy. More controlled clinical trials are warranted for a cumulative progress in this direction.

\section{References}

1. Zumla A, Nahid P, Cole ST (2013) Advances in the development of new tuberculosis drugs and treatment regimens. Nat Rev Drug Discov 12: 388-404. [PubMed]

2. World Health Organisation (2012) Totally Drug- Resistant TB: a WHO consultation on the diagnostic definition and treatment options.

3. Falzon D, Jaramillo E, Schünemann HJ, Arentz M, Bauer M, et al. (2011) WHO guidelines for the programmatic management of drug resistant tuberculosis: 2011 update. Eur Resp Jour 38: 516-528. [PubMed]

4. Jacobson KR, Tierney DB, Jeon CY, Mitnick CD, Murray MB (2010) Treatment outcomes among patients with extensively drug resistant tuberculosis: systematic review and meta- analysis. Clin Infect Dis 51: 6-14. [PubMed]

5. Uhlin M, Andersson J, Zumla A, Maeurer M (2012) Adjunct immunotherapies for tuberculosis. J Infect Dis 205: S325-334. [PubMed]

6. Nandi B, Behar SM (2011) Regulation of neutrophils by interferon gamma limits lung inflammation during tuberculosis infection J Exp Med 11: 289-262. [PubMed]

7. Pittinger MF, Martin BJ (2004) Mesenchymal stem cells and their potential as cardiac therapeutics. Cir Res 95: 9-20. [PubMed]

8. Horwitz EM, Le Blanc K, Dominici M, Mueller I, Slaper-Cortenbach I, et al (2005) Clarification of the nomenclature for MSC: The International Society for Cellular Therapy position statement. Cytotherapy 7: 393-395. [PubMed]

9. Dominci M, Le Blanc K, Mueller I, Slaper-Cotenbach I, Marinin F, et al. (2006) Minimal criteria for defining multipotent mesenchymal stromal cells. The International Society for Cellular Therapy position statement. Cytotherapy 8 : 315-317. [PubMed]

10. Chelluri LK, Prasad CE, Preethi V, Gokhale AGK, Vasantha A, et al. (2010) Tumor necrosis factor (alpha) and Interferon-gamma in allogenic mixed cultures of hematopoietic and non-hematopoietic cell-cell interactions; TB a case study. Journal of Infectious Diseases.

11. Pittenger MF, Mackay AM, Beck SC, Jaiswal RK, Douglas R, et al. (1999) Multilineage potential of adult human mesenchymal stem cells. Science 284 : 143-147. [PubMed]

12. Reyes MT, Lund T, Lenvik D, Agular L, Koodle L, et al. (2001) Purification and ex-vivo expansion of postnatal human marrow mesodermal progenitor cells. Blood 98: 2615-2625. [PubMed]

13. Gandhi NR, Moll A, Sturm AW, Pawinski R, Govender T, et al. (2006) Extensively drug resistant tuberculosis as a cause of death in patient coinfected with tuberculosis and HIV in rural area of South Africa. Lancet 368: 1575-1580. [PubMed]

14. Gao XF, Yang ZW, Li J (2011) Adjunctive therapy with interferon- gamma for the treatment of pulmonary tuberculosis: a systematic review. Int $\mathrm{J}$ Infect Dis 15: e594-e600. [PubMed]

15. Bonfield TL (2010) Adult Mesenchymal stem cells: An Innovative therapeutic for lung diseases. Discov Med 9: 337-345. [PubMed]

16. Dennis JE, Cohen N, Goldberg VM, Caplan Al (2004) Targeted delivery of progenitor cells for cartilage repair. J Orthop Res 22: 735-741. [PubMed]

17. Lennon BP, Haynesworth SE, Young RG, Dennis JE, Caplan Al (1995) A chemically defined medium supports in vitro proliferation and maintains the osteochondral potential of rat marrow derived mesenchymal stem cells. Exp Cell Res 219: 211-222. [PubMed]

18. Caplan Al (2009) Why MSC are's therapeutic? New data: new insight. J Pathol 217: 318-324. [PubMed]

19. Iyer SS, Rojas M (2008) Anti- inflammatory effects of mesenchymal stem cells: novel concept for future therapies. Expert Opin Biol Ther 8: 569-581. [PubMed]

20. Oritz LA, Gambelli F, Mcbride C, GauppD, Baddoo M, et al. (2003) Mesenchymal stem cells engraftment in lung is enhanced in response to bleomycin exposure and ameliorates its fibrotic effects. Proc Natl Acad Sci USA 100: 8407-8411. [PubMed]

21. Zhao F, Zhang YF, Lui YG, Zhou JJ, Li ZK, et al (2008) Therapeutic effects of bone marrow derived mesenchymal stem cells engraftment on Ibleomycin induced lung injury in rats. Transplant Proc 40: 1700-1705. [PubMed]

22. Moodley Y, Atienza D, Manuelpillai U, Samuel CS, Tchongue J, et al. (2009) Human umbilical cord mesenchymal stem cells engraftment on bleomycin induced lung injury. Am J Pathol 175: 303-313. [PubMed]

23. Nemeth K, Leelahavanichkul A, Yuen PS, Mayer B, Parmelee A, et al. (2009) Bone marrow stem cells attenuate sepsis via prostaglandin E2 dependent reprogramming of host macrophages to increase their interleukin-10 production. Nat Med 15: 42-49. [PubMed]

24. Gonzalez-Rey E, Anderson P, Gonzalez MA, Rico L, Buscher D, et al. (2009) Human adult stem cells derived from adipose tissue protect against experimental colitis and sepsis. Gut 58: 929-939. [PubMed]

25. Prockop DJ (1997) Marrow stromal cells as stem cells for non haematopeitic tissues. Science 276: 71-74. [PubMed]

26. Shi Y, Su J, Robverts Al, Shou P, Robson A, et al. (2012) How mesenchymal stem cells interact with tissue immune responses. Trends Immunol 33: 136143. [PubMed]

27. lyer SS, Co C, Rojas M (2009) Mesenchymal stem cells and inflammatory lung diseases. Panminerva Medica 51: 5-16. [PubMed]

28. Sato k, ET AL (2007) Nitric oxide plays a critical role in suppression of T cell proliferation by mesenchymal stem cells. Blood 109: 228-234. [PubMed]

29. Guyot-Revol V, Innes JA, Hackforth S, Hinks T, Lalvani A (2006) Regulatory T cells are expanded in blood and disease sites in patients with tuberculosis. Am J Resp Crit Care Med 173: 803-810. [PubMed]

30. Scott-Browne JP, Shafiani S, Tucker-Heard G, Ishida-Tsubota K, Fontenot JD, et al. (2007) Expansion and function of Foxp-3 expressing T regulatory cells during tuberculosis. J Exp Med 204: 2159-2169. [PubMed]

31. Ren G, Zhang L, Zhao X, Xu G, Zhang Y, et al. (2008) Mesenchymal stem cell mediated immunosuppression occurs via concerted action of chemokines and nitric oxide. Cell Stem Cell 2: 141-150. [PubMed]

32. Raghuvanshi S, Sharma P, Singh S, Kaer LV, Das G (2010) Mycobacterium tuberculosis evades host immunity by recruiting mesenchymal stem cells. Proc Natl Acad Sci 107: 21653-21658. [PubMed]

33. Krasnodembskaya A, Song Y, Fang X, Gupta N, Serikov V, et al. (2010) Antibacterial effect of human mesenchymal stem cells is mediated in part from secretion of the antimicrobial peptide LL-37. Stem cells 28: 2229-2238. [PubMed]

34. Skrahin A, Ahmed RK, Ferrara G, Rane L, Poiret T, et al. (2014) Autologous mesenchymal stromal cell infusion as adjunct treatment in patients with multidrug and extensively drug-resistant tuberculosis: an open-label phase 1 safety trial. Lancet Respir Med 2: 108-122. [PubMed]

35. Javaregowda PK, Yoon JW, Jang G (2013) Roles of mesenchymal stem cells (MSCs) in bacterial diseases. J Biomed Res 14: 184-194.

36. Tropea KA, Leder E, Aslam M, Lau AN, Raiser DM, et al. (2012) Bronchioalveolar stem cells increase after mesenchymal stromal cell treatment in a mouse model of bronchopulmonary dysplasia. Am J Physiol Lung Cell Mol Physiol 302: L829-L837. [PubMed]

37. Hare JM, Fishman JE, Gerstenblith G, et al. (2012) Comparison of allogeneic vs. autologous bone- marrow derived mesenchymal stem cells delivered by trans-endocardial injection in patients with ischemic cardiomyopathy: the POSEIDON randomized trial. J Am Med Assoc 308: 2369-2379. 